\title{
Induction treatment strategy in multiple sclerosis: a review of past experiences and future perspectives
}

\author{
Serena Ruggieri ${ }^{1,2}$, Simona Pontecorvo ${ }^{1}$, Carla Tortorella ${ }^{2}$ and Claudio Gasperini ${ }^{2 *}$ (1)
}

\begin{abstract}
The scenario of multiple sclerosis (MS) treatment has changed profoundly in recent decades. In this setting, one of two strategies is usually used: escalation or induction. The first involves a pyramid of possible treatments of increasing efficacy (but also increasing safety risks) that are introduced progressively as needed. The induction strategy, on the other hand, immediately pursues higher efficacy, since drugs with a higher risk profile are used from the outset. Understanding which of these treatment strategies is the more suitable for a given patient is the first step in the therapeutic decision-making process. Prognostic factors evaluated on the basis of the clinical presentation and any disease activity on magnetic resonance imaging (MRI) should guide and help clinicians in making their choices.

Even though the pathogenesis of MS is not yet completely understood, specific pathological changes are known to occur in the adaptive and innate immune system over the course of the disease. To date, treatment has been based mainly on two drugs, mitoxantrone and cyclophosphamide, autologous haematopoietic stem cell therapy (within clinical trial setting), but new compounds are now emerging. Among the new treatments, alemtuzumab and cladribine appear to be valid candidates as induction drugs.

In this review we provide an overview of induction strategies based on literature evidence and our own past experiences, providing descriptions of clinical cases. We also outline the future perspectives in this field.
\end{abstract}

Keywords: Induction strategy, Highly active multiple sclerosis, Immunosuppression

\section{Background}

Multiple sclerosis (MS) is a progressive and highly debilitating disease that places a high burden both on individual patients and on society. Current disease-modifying therapies (DMTs) for MS are effective for decreasing relapses and slowing progression, but they fail to adequately address a series of needs in terms of treatment, disability avoidance and the importance of considering patients' own priorities in order to improve their lives. Goal assessment and individualization of therapy should thus take into account current disease activity and disability, the patient's lifestyle and preferred treatment administration route, as well as whether or not

\footnotetext{
* Correspondence: c.gasperini@libero.it

2Department of Neurosciences, S Camillo-Forlanini Hospital, Circonvallazione Gianicolense, 87, 00152 Rome, Italy

Full list of author information is available at the end of the article
}

he/she can self-treat or instead needs therapy to be delivered by a healthcare professional.

Accordingly, in neurological clinical practice, as in other medical fields, efforts are being made to develop an "individualized treatment" approach. The challenge faced by specialists in the field of MS, in particular, is to identify the most effective drug and strategy for each individual patient at each phase of the disease, that is to choose a therapeutic approach which has necessary efficacy to treat the actual disease. In recent decades, two strategies for managing MS therapies have been recognized: escalation (Fig. 1a), where treatment starts with lower-risk, lower-efficacy DMTs and only moves on to more aggressive treatments if the ongoing approach fails; and induction (Fig. 1b), which can be defined as treatment based on the use of high-efficacy DMTs with a sustained, long-term biological effect in treatment-naïve patients. Induction therapy is based on the early use of 


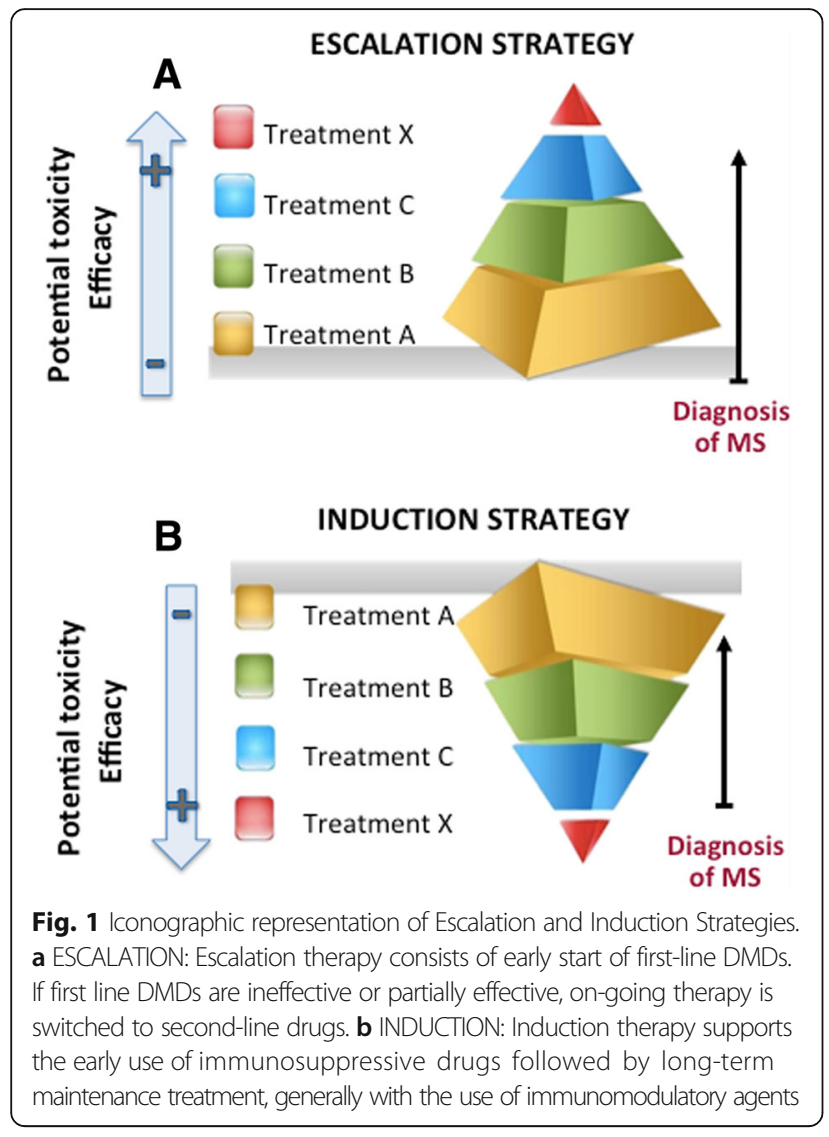

immunosuppressive drugs followed by long-term maintenance treatment, generally with immunomodulatory agents.

The concept of induction strategy takes his first steps in oncology and rheumatology practice where, one drug followed by long-term maintenance treatment with a different drug, has proved its utility on long term outcomes [1]. Accordingly to different oncology expertise this scheme has been used from head and neck tumors, to lung cancer and leaukemias. For istance an optimal use of the same antileukemic agents (developed from the 1950 s through the 1980s), along with a rigorous application of prognostic factors for risk-directed therapy in clinical trials, resulted in a continuos improvement in treatment outcome. The goal of remission-induction therapy was to eradicate more than $99 \%$ of the initial load of leukemia cells and to reconstitute normal hematopoiesis and a normal performance status. When normal hematopoiesis is restored, patients in remission become candidates for intensification (consolidation) therapy [2].

During the BeSt study, a multicentre randomized single blind trial that integrated targeted treatment and tight control with a comparison of 4 treatment strategies in recent onset rheumatoid arthritis (RA), authors showed that early and maintained targeted treatment had functional and radiological benefits over the first 7 years of disease history and can even include drug tapering and (partial, temporary or permanent) discontinuation [3].

The span of available treatment that can be used as induction therapy is somewhat narrow compared to current DMTs. In the last decade's mitoxantrone (MTX), cyclophosphamides (Cys) and autologous haematopoietic stem cell therapy (within clinical trial setting) have been used with this aim. New and recently approved treatment whose mechanism of actions let to define them as possibly induction therapy may include alemtuzumab (AZB) and cladribine. According to their label, even fingolimod and natalizumab, can be employed as treatment options in naïve patients if the disease course is considered particularly aggressive. Despite the high effectiveness of both treatment they should not be chosen as induction therapies because their quickly reversible mechanisms of action predispose patients to a return of disease activity following treatment discontinuation (Fig. 2) [4].

Although the definition of induction or escalation strategy neatly define a practical approach, our choice of treatment, regardless of the strategy chosen, should nevertheless be based on three simple questions: Why? When? How? [5].

In this review we focus on induction strategy, looking at the latest literature evidences and trying to answer to the above questions in the light of our own clinical experiences.

\section{Rationale and pathological background}

The induction strategy concept in MS is based on knowledge of the interplay between two main aspects of the disease, in short, on knowledge of the immunological mechanisms underlying MS pathology and the way in which, at different levels, these influence the phenotypical expression of the disease.

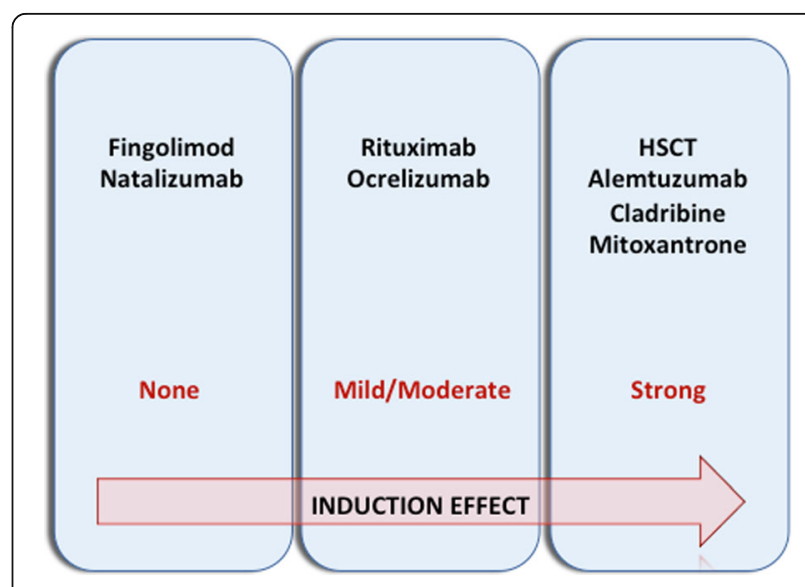

Fig. 2 Potential inductive effect of DMDs in Multiple Sclerosis 
MS is a complex disease in whose development genetic factors, immunological components and environmental influences all play a role. MS pathological changes occur in the adaptive and innate immune system over the course of the disease. Although the disease trigger is still unknown, it has been established that T-cells attack myelin components, including myelin basic protein, myelin oligodendrocyte glycoprotein and proteolipid protein. But the pathological mechanisms consist of more than just an autoimmune attack against a single target. In MS, T-cells react to an initial myelin epitope displayed by an antigen-presenting cell (such as a dendritic cell) that is linked to a particular major histocompatibility complex protein. After symptoms decline, persistent inflammation may induce a new wave of as yet unactivated T-cells to enter the central nervous system (CNS), where, in the presence of a co-stimulatory signal, they become primed to new epitopes - a phenomenon named "epitope spreading" [6]. Immune infiltration from the periphery constitutes a prominent feature of early-stage MS and T-cells (CD 4+, CD8+, natural killer) can enter the CNS through different sites (meningeal blood vessels, subarachnoid space, choroid plexus). These cells, in the presence of an activated CNS-resident immune system, promote demyelination through direct cell contact-dependent mechanisms and through the action of soluble inflammatory and neurotoxic factors. Later on in the disease course immune cell infiltration decreases, perhaps due to exhaustion of adaptive immune cells as a result of chronic antigen exposure. Thus, chronic CNS inflammation may help to sustain the neurodegenerative process through the action of cells that have entered or are already resident within the CNS, along with oxidative stress responses, energy deficiencies, ionic imbalances and the failure of neuroprotective and regenerative mechanisms [7].

This immunological cascade, here extremely simplified, may be seen as a two-stage process that can explain the phenotypic expression of the disease. This consists of an initial phase in which relapses represent the main aspects of the illness and patients show a mild level of disability, and a second phase in which clinicians usually observe a slow but irreversible progression of the disability, which gradually becomes more severe [8]. On the basis of this evidence, the rationale behind induction strategy is thus to influence the initial inflammatory phases, so as to avoid the subsequent chronic phase that helps to maintain the neurodegenerative process and leads to irreversible disability. The advantage of induction therapy can be seen as a "resetting" of the immunological system to prevent the phenomenon of epitope spreading which can result in better control of disease activity. In order to obtain this effect it is fundamental to choose a drug with a specific mechanism of action, i.e. one that has the capacity to bring about near to total immune suppression followed by gradual restoration of the immune cell population through a modified pathway. Since in our treatment landscape we still do not have drugs able to modify the neurodegenerative component of disease, one would want to time treatment to coincide with the presence of ongoing inflammation. This concept has been defined has "treatment window" [9] in which we can detect the early opportunity to influence the accumulation of irreversible long-term damage, choosing high-efficacy therapy that targets both focal and diffuse pathology thus having a favorable impact on long-term outcomes [10]. However induction strategy, and particularly those drugs that are considered to be good candidate to fall in this category, cannot be used in every patients due to safety concerns that are often associated with their use. The high efficacy of these treatments needs to be weighed individually by their relatively higher risk profile.

\section{Selection of patients}

Given the relatively greater risks inherent in the use of high-efficacy DMTs, patients who may potentially benefit from early use of these treatments must be selected with great care. Indeed, in view of evidence that high-efficacy therapies carry greater safety risks, the induction strategy has generally been reserved for patients whose disease is very active and aggressive in terms of clinical relapses or disease activity on magnetic resonance imaging (MRI). Patients falling into this category are defined "highly active", a label that not only indicates the presence of very active and aggressive disease at the time of observation, but may also indicate the presence of concomitant negative prognostic factors.

The main problem in pursuing an induction strategy is that, by definition, it is more effective in the early stage of disease, but giving the chronic and long lasting nature of MS, understand and label the disease at its onset is particularly challenging. For this reason a great effort has been put to define those clinical and paraclinical characteristics that allow us to define a patient as highly active or not.

Longterm natural history studies [11] provide precious information about negative prognostic factor presenting early at onset or during follow-up and infer that late disability can be predicted on the basis these factor, though helping in identify patients at risk of a more aggressive disease course [12] (Table 1). An epidemiological study assessed on a database of 5891 patients with MS, the proportion of patients that could be defined as affected by "aggressive MS" according to an Expanded Disability Status Scale (EDSS) of 6 (reached in 5 years or before the age of 40) or development of secondary progressive MS (SPMS). Interestingly out of a percentage of $4-14 \%$ 
Table 1 Negative Prognostic factors in naïve MS patients [12]

\begin{tabular}{|c|c|c|}
\hline \multirow[t]{4}{*}{ Clinical Feature } & Demographics & $\begin{array}{l}\text { - Male sex } \\
\text { - Older age (> } 40 \text { years) at onset }\end{array}$ \\
\hline & Relapse severity & $\begin{array}{l}\cdot \geq 1 \text {-point change on EDSS, } \geq 2 \text {-point change on any individual functional } \\
\text { system, or } \geq 1 \text {-point change on any two functional systems } \\
\text { - Steroid requirement } \\
\cdot \text { Hospitalization }\end{array}$ \\
\hline & Relapse frequency & $\begin{array}{l}\text { - Frequent relapses in the first } 2 \text { years } \\
\text { - Short time interval between relapses }\end{array}$ \\
\hline & Disease Course & - Rapid increase of disability (e.g EDSS score $\geq 4$ ) in 12 months \\
\hline MRI charatersitics & & $\begin{array}{l}\text { - High T2 lesion load } \\
\text { - More than two gadolinium-enhancing lesions } \\
\text { - Presence of T1 lesions ('black holes') } \\
\text { - Infratentorial lesions }\end{array}$ \\
\hline
\end{tabular}

of patients falling into the pervious definition, the majority of these patients had relapsingonset MS and were, for this reason, possibly candidate to disease modifying treatment [13]. Usually patients with frequent relapses at the time of diagnosis and those who accumulate a large number of focal lesions detected on T2-weighted MRI sequences or gadolinium (Gd)-enhancing lesions within the first five years become disabled more quickly than patients who do not [14].

Taking into account the heterogeneous pathological processes underlying MS and the complexity of the disease, a composite evaluation that considers several measures might be able to furnish a more comprehensive estimation of disease activity. Despite the evident difficulties in selecting univocally patients with precise characteristics and negative prognostic factors at beginning of the disease, the following main criteria for identifying potential good candidate for induction treatment have been recognized among others: a younger age (ideally under 40 years old), a pure relapsing-remitting (RR) form of MS, at least two relapses within the previous 12 months, a severe relapse resulting in an EDSS score $\geq$ 4 or a score increase of two or more points within the previous 12 months, and the presence of two or more additional Gd-enhancing lesions on a recent MRI scan [5].

This can be emphasized with a slogan adopted from an awareness campaign used in ischemic stroke pathological studies: "time is brain". Thus we would affirm that "timing" matters in MS and indicate that the optimal timing would be in the evidence of significant clinical and MRI inflammatory activity without acquired irreversible disability [15].

\section{Treatment choices: Past and present experiences}

Several clinical trials have investigated potential induction treatment drugs. In the first MS treatment era, MTX and Cys, two immunosuppressant drugs, were widely used. In this treatment approach, even though the latter has been used only off-label or in clinical trials, due to the lack of adequate phase III studies, it has not been formally approved for treatment of MS.

MTX is an anti-neoplastic anthracenedione derivative that is related to the class of anthracyclines. It is usually administered intravenously at a dose of $12 \mathrm{mg} / \mathrm{m}^{2}$ every three months until a maximum total cumulative dose of $140 \mathrm{mg} / \mathrm{m}^{2}$ has been reached, but this therapeutic regimen may vary. In recent years, use of MTX has decreased due to the risk of severe adverse events [16].

Following a pivotal trial [17] and an observational study [18] in highly active relapsing-remitting MS (RRMS) and SPMS patients, MTX was tested in a three-year clinical and MRI study in aggressive MS patients defined as those who had experienced two or more relapses in the previous 12 months and shown one or more Gd-enhancing MRI lesion [19]. One hundred and nine patients were randomized into two groups: 54 patients received MTX monthly $\left(12 \mathrm{mg} / \mathrm{m}^{2}\right.$; maximum $20 \mathrm{mg}$ ) combined with $1 \mathrm{~g}$ of MP for six months followed by interferon (IFN) for the last 27 months; the other 55 patients received IFN-beta-1b (250 mg subcutaneously every other day) for three years combined with $1 \mathrm{~g}$ of MP monthly for the first six months. The primary endpoint was the time to worsen by at least one EDSS point, confirmed at three months. The two patient groups had comparable clinical and demographic features at inclusion. The patients underwent a complete neurological examination every three months and spin-echo MRI at inclusion and at months 9, 24 and 36. The time to worsen by at least one EDSS point, confirmed at 3 months, was delayed by 18 months in the MTX group compared with the IFN group $(p<0.012)$. The three-year risk of worsening disability was reduced by $65 \%$ in the MTX group relative to the IFN group $(11.8 \%$ vs $33.6 \%)$. The proportion of patients who remained relapse-free was increased in the MTX group $(p<0.008)$. The MTX patients had a $61.7 \%$ reduced relapse rate, a reduced number of Gd-enhancing lesions at 
month 9, and a slower accumulation of new T2 lesions at each time point [9].

Another controlled study [20], by a different research group, enrolled 40 RRMS patients with 1-15 Gd-enhancing lesions on MRI and EDSS scores of 0-6.5. The patients were randomized to receive either short-term induction therapy with MTX (three $12 \mathrm{mg} / \mathrm{m}^{2}$ infusions, one per month) followed by 12 months of daily glatiramer acetate (GA) therapy at $20 \mathrm{mg} /$ day subcutaneously for a total of 15 months $(n=21)$, or GA at $20 \mathrm{mg} /$ day for 15 months $(n=19)$. MRI scans were performed at months 6, 9, 12 and 15. The MTX-GA induction treatment led to an $89 \%$ greater reduction [relative risk = $0.11, p=0.0001]$ in the number of Gd-enhancing lesions at months 6 and 9 compared to GA alone and a 70\% greater reduction (relative risk $=0.30, p=0.0147$ ) at months 12 and 15. Mean relapse rates were 0.16 and 0.32 in the MTX-GA and GA group respectively. During the 15 -month study, $81 \%$ of MTX-GA patients and $79 \%$ of GA patients remained relapse free [20].

Due to increasing concerns regarding the safety profile of MTX, in 2001 a French multicenter prospective study of a large cohort of MS patients was set up. This study entailed annual updates for at least the first five years after initiation of MTX therapy. In total, 802 patients were treated with a mean cumulative dose of $72 \mathrm{mg} / \mathrm{m}^{2}$. They were then followed up through clinical, hematological and echocardiographic examinations for the five years following the start of the treatment. One of the 802 patients $(0.1 \%)$ developed acute congestive heart failure. Two cases of therapy-related leukemia (0.25\%) were detected 20 months after starting MTX (one patient died while a remission of 8 years was reported for the other). Of the 317 women treated before the age of $45,17.3 \%$ developed persistent age-dependent amenorrhea $(5.4 \%$ before 35 years of age, $30.7 \%$ after 35 years of age) [21]. The risk of acute leukemia has also been estimated by other research groups, which reported rates higher than [22] or similar to [23] that reported in the French cohort.

Cys is an alkylating chemotherapeutic agent related to nitrogen mustard that binds to DNA and interferes with mitosis and cell replication, and hence targets rapidly dividing cells [24]. Treatment regimens vary: one consists of intermittent pulse therapy given monthly or bimonthly over a one- to three-year period, administered intravenously at an adjusted dose to obtain a leucopoenia target, or at a fixed dose [25]; with another, infusion of $200 \mathrm{mg} / \mathrm{kg}$ of Cys over four days to induce profound leucopenia is followed by temporary use of filgastrim (granulocyte-colony stimulating factor) to hasten endogenous granulocyte recovery [26].

One of the first trials using Cys in MS was an open-label study with intravenous Cys $\left(500 \mathrm{mg} / \mathrm{m}^{2}\right.$ for five days followed by maintenance therapy) in $17 \mathrm{pa}$ tients with fulminant MS, defined as worsening of more than one and a half points on the EDSS for more than three months. In 24 months, $69 \%$ of patients were clinically stable or improved [27].

In a two-year randomized trial, researchers compared the effect of 12 months of induction therapy with Cys followed by IFN-beta versus IFN-beta alone. The authors enrolled 20 active RRMS patients, defined as those who had had at least one relapse and one Gd-enhancing lesion detected on MRI during the previous year. Patients were randomized to receive Cys monthly (to induce a leucopoenia below $1000 \times \mathrm{mm}^{3}$ ) plus methylprednisolone (MP) $1 \mathrm{~g}$ for 12 months followed by IFN-beta for a further 12 months (cy group) or IFN-beta alone for two years (IFN-beta group). The authors observed a reduction in the relapse rate and in the number of Gd-enhancing lesions at the end of follow-up (24 months). Relapse-free patients at the end of the second year amounted to $80 \%$ in the Cys group versus $40 \%$ in the IFN-beta group [25].

A retrospective review of a closely followed population of 32 MS patients treated with high-dose Cys $(200 \mathrm{mg} /$ $\mathrm{kg}$ intravenous infusion over four days) followed by maintenance therapy with GA was performed in a US center with the aim of assessing the safety and efficacy of this treatment regimen. The annualized relapse rate (ARR) was reduced from 1.37 in the two years prior to treatment to 0.27 over a mean post-treatment follow-up period of 14 months (range 0.5-33.8). The mean number of Gd-enhanced lesions was reduced from $0.86( \pm$ 1.6) at baseline to 0 at 12 months and $0.08( \pm 0.28)$ at $15-24$ months. In total, $55 \%$ of patients had no evidence of disease activity at follow-up. Infectious complications occurred in $47 \%$ with no long-term morbidity and no deaths [16].

Long-term use of Cys is limited by its well-known bladder toxicity, which is manifested as hemorrhagic cystitis and bladder cancer. This event may occur a few years after cessation of the therapy. Hemorrhagic cystitis can be seen in up to $4.5 \%$ of MS patients treated with Cys [24]. The risk of bladder cancer appears to increase as a function of the total dose. In a retrospective study of 2351 patients with MS, two women and five men $(0.29 \%)$ had bladder cancer. In the 850 chronically catheterized patients, the incidence was $0.7 \%$. In the subgroup of 70 patients previously treated with Cys, five chronically catheterized patients (5.7\%) had bladder cancer. The mean time from the last dose of Cys until the diagnosis of neoplasm was 5.8 years (range $3-10$ years). No other Cys-induced tumors emerged [28].

Intense immunosuppression followed by autologous hematopoietic stem cell transplantation (aHSCT) has been an option during the last twenty years for those patients that experience accumulated disability regardless 
of treatment or rapidly worsening or fulminant MS, with frequent relapses. The aim of aHSCT treatment is the total depletion of autoreactive cells, followed by the infusion of autologous hematopoietic stem cells to reestablish and reset the aberrant hematolymphopoietic system [29]. Approximately 800 patients with MS have been treated with aHSCT worldwide, always in clinical trial settings.

Initial studies selected more-severely disabled patients to establish the safety of the procedure, but didn't achieve good results in terms of efficacy, and were characterize by an high mortality rate up to $5 \%$. Lately, treatment-related mortality rates decreased from $7.3 \%$ for the period 1995-2000 to $1.3 \%$ during the period 2001-2007 [30]. This might be seen as a consequence of better selection of patients who were not inexorably disabled and who had less-advanced disease, as well as to general advances in post-transplant care [12]. Different transplant procedures are currently in use, but given a lack of comparative studies no evidence exists for one regimen being superior to another. The ASTIMS trial, a multicenter, phase II, randomized trial including 21 patients with SP or RRMS, compared the impact of aHSCT vs MTX on disease activity measured by MRI. aHSCT reduced by $79 \%$ the number of new T2 lesions as compared to MTX (rate ratio 0.21, $p=0.00016$ ) and Gd + lesions (rate ratio $=0.19,95 \%$ CI $0.09-0.41, p<0.0001$ ) as well as the annualized relapse rate. No treatment group difference was detected in the progression of disability. Early adverse events were considered as expected and occurred at least in $80 \%$ of treated cases. SAE occurred in the aHSCT arm only and resolved without sequelae [31]. Despite these encouraging results, and improvement of clinical expertise, this type of treatment need to be performed only in selected centers with known neurological and hematological expertise. Furthermore whether the procedure is really effective in modifying the progressive course of the disease, to assess long-term efficacy and safety deserves additional evalutation in phase III trials.

\section{Clinical experience}

Here we present two illustrative clinical cases that outstand the concept of different outcomes accordingly to different timing in choosing treatment. Of course the cases below refers to a past treatment era and probably nowadays our approach would be diverse. However our aim is to present a clinical scenario, despite of the drug used, that can exemplify the concept of "window of opportunity". Thus regardless of date and consequent treatment scheme applied, the clinical cases below represent an opportunity to reflect on the consequence of a particular choice given a particular time during the disease course. We can easily transfer these clinical presentations to our every day clinical practice in the present years and challenge ourselves on which compound we might chose giving the current enriched treatment armamentarium. Indeed, both cases are characterized by a severe clinical onset and poor prognostic factors at onset. In the first case a strong immunosuppression with MTX was started immediately after diagnosis of MS. In the second case - on the contrary - i.m. IFNbeta 1a was started and then MTX was used as rescue therapy.

Case report 1 A 34-year-old right-handed woman was admitted to hospital on the May 31st, 2006 with acute onset of dizziness, visual impairment in her right eye and tingling in all four limbs. Her history included a recent episode (February 2006, three months earlier) of non-painful visual field impairment in her right eye. She was diagnosed by an ophthalmologist with optic neuritis $(\mathrm{ON})$ and treated with oral prednisolone, obtaining complete regression of the symptoms. Her general examination was otherwise normal. A cerebral CT scan showed a hypodense white matter lesion in the left temporal lobe (maximum diameter: $1 \mathrm{~mm}$ ) and one in the left frontal lobe (maximum diameter: $7 \mathrm{~mm}$ ). On MRI multiple areas in the white matter (the periventricular area, corpus callosum, bilateral cerebellar peduncles, centrum semiovale bilaterally) had an appearance consistent with demyelination; most of these areas appeared confluent. At least eight lesions showed contrast enhancement after Gd injection.

The patient was admitted to the neurology ward where a neurological examination revealed horizontal and vertical diplopia, mild left lower motor neuron 7th cranial nerve palsy, pyramidal weakness of the left arm[(Medical Research Council (MRC) scale scores of $4 / 5$ proximally and 2/3 distally], dysmetria in the four limbs (left > right), and brisk reflexes with a left extensor plantar reflex; sensation could not be evaluated. Gait was not evaluated because of recent stem cell transplantation for aseptic necrosis of femoral head (of uncertain etiology). Autoimmune blood screening was normal, as were sensory and motor evoked potentials, and thorax and abdomen CT scans.

Oligoclonal bands were detected in the cerebrospinal fluid (CSF), with fewer bands in the serum, consistent with intrathecal synthesis of immunoglobulin. CSF analysis was negative for enterovirus, herpes simplex virus types 1 and 2, varicella zoster virus and John Cunningham (JC) virus on polymerase chain reaction. CSF bacterial and tuberculosis culture was negative. Serology was negative for HIV, HTLV, Borrelia, syphilis and JC virus. The patient had normal full blood count values and renal, liver and thyroid function values. Vitamin B12 and folate levels were also normal. Antibody test 
results (aquaporin, anti-double stranded DNA, ENA and anti-neutrophil cytoplasmic antibody) were normal. MRI of the brain (with single-dose Gd) performed during hospitalization confirmed the presence, in the white matter, of multiple areas consistent with demyelination; a brainstem and a cerebellar lesion each showed Gd enhancement. MRI of the cervical spine showed a demyelinating lesion at C5-C6 level. The patient was diagnosed with MS and treated with intravenous methyl prednisolone (IVMP), $1 \mathrm{~g}$ per day for five days, which produced a weak improvement of the symptoms. Intraorbital corticosteroids were injected to reduce optic nerve inflammation, with minimal results.

After informed discussion with the patient and her family about starting her on a DMT, an immunosuppressive drug was suggested. In July 2006 the patient started receiving MTX $10 \mathrm{mg} / \mathrm{m}^{2}$ bimonthly. No side effects were reported. In September 2006 an MRI showed new T2 lesions in the posterior left brainstem and in the parietal lobe, even though no new symptoms were reported; these possibly dated back to the previous July, when MTX had just been started. Thereafter, complete clinical and radiological stabilization was achieved. In September 2008 the patient became pregnant. Her pregnancy was unremarkable and she gave birth to a healthy child.

In September 2009 patient started treatment with high-dose IFN-beta-1a. She has had no further relapses; to date, clinical and radiological follow-up continues to show complete stabilization of her condition.

Case report 2 A 23-year-old man was admitted to our clinic in November 2004 due to subacute onset of dysarthria and cerebellar ataxia. A brain and spine MRI was performed and showed multiple areas of high signal in the white matter, consistent with demyelination (the periventricular area, corpus callosum, bilateral cerebellar peduncles, bilateral centrum semiovale, cerebellar hemispheres, and spinal levels C2-C3 and C3-C4 and C5-C6). A brainstem lesion showed Gd enhancement. Neurological examination detected horizontal and vertical diplopia, rotating and horizontal pendular nystagmus, pyramidal weakness of the left arm (MRC scale score 4/ 5), reduced sensitivity below T2 level, dysmetria (left > right), hypotonia, brisk reflexes with bilateral extensor plantar response, ataxic gait of both cerebellar and sensory origin, neurogenic bladder. Full autoimmune blood screening was normal and the patient was diagnosed with MS and started on IFN-beta-1a intramuscularly. Follow-up MRI in May 2005 showed new T2 lesions in the frontal white matter, and a new T2 lesion located at spinal level D3. In September 2005 the patient experienced blurred vision and was treated with IVMP $1 \mathrm{~g}$ per day for five days.
In July 2006 the patient experienced acute bladder retention and sensory impairment below spinal level T3. He was administered IVMP $1 \mathrm{~g}$ per day for five days.

In December 2006 the patient presented a new medullary syndrome. MRI showed a new T11-L1 spinal lesion with contrast enhancement.

IFN was stopped and MTX, bimonthly, was started in January 2007. After six infusions of MTX the cerebellar syndrome improved dramatically, and this improvement was maintained over time. MTX was administered for two years and stopped when the maximum dose allowed was reached. In March 2009 GA was prescribed as maintenance therapy.

In December 2010 a clinical relapse was reported, consisting of an acute right pyramidal syndrome. This was treated with corticosteroids. Over the following six months, the patient experienced two episodes of $\mathrm{ON}$, resulting in permanent reduction of visual acuity bilaterally. On a brain MRI scan performed in June 2010, supratentorial lesions were found to be increased.

Treatment with GA was stopped and the patient was switched to fingolimod as a second-line therapy. Treatment with natalizumab was ruled out due to the patient's positive JC virus antibody status.

Over the subsequent years this patient has experienced a slow clinical worsening characterized by gait and visual acuity impairment.

\section{Treatment choices: Future perspectives}

The past 10 years have seen the introduction of several new compounds for treating MS, characterized by different mechanisms of action and different administration routes. Among these newly available or soon-to-be available treatments, we can identify two drugs that have emerged as induction treatments, whose administration should be followed by the use of already established and less aggressive medications.

AZB is a monoclonal antibody that recognizes the CD52 epitope located on the surface of mononuclear cells; CD52 is primarily expressed on $\mathrm{CD} 4^{+}$and $\mathrm{CD} 8^{+} \mathrm{T}$ lymphocytes, B-cells, and monocytes. Administered alone (12 mg per day, intravenously, for five days, with a re-treatment at the same dose for three days one year later), AZB has a profound and sustained effect on the inflammatory process and can cause lasting global immunosuppression.

Upon binding to CD52, AZB rapidly and effectively eliminates circulating CD52 cells via antibody and complement-mediated depletion. Soon after administration of the compound, circulating lymphocytes are virtually undetectable in peripheral blood. Subsequently, the adaptive immune system is reconstituted from precursor cells or mature cells that have escaped depletion. The dynamics of this repopulation differ according to cell 
lineages: monocytes and B-cells are the first to repopulate in the peripheral blood, approximately 3-6 months after treatment. T-cells, particularly $\mathrm{CD} 4^{+}$cells, reappear more slowly, with normal levels once again detectable after 2-3 years and pretreatment levels only after almost five years have elapsed [32].

In a three-year phase II trial in early MS, annual AZB infusion was compared with administration of an IFN-beta-1a preparation [33]. Patients enrolled in this study had early, active MS, and were naïve to prior immunotherapy for MS other than steroids. AZB treatment reduced disability progression, improved disability scores, and decreased relapse rate by $70 \%$ compared with IFN-beta-1a, but this trial was interrupted early as three patients developed idiopathic thrombocytopenia, and one of them died. Post hoc analyses showed that, at year 3, $73 \%$ of the patients treated with AZB were free from clinical disease activity, a state defined as absence of six-month sustained accumulation of disability and relapse, compared with $43 \%$ in the IFN-beta-1a group (HR, 0.33; $p<0.0001$ ) [34].

In a phase III trial, the superiority of AZB over IFN-beta on relapse rates was confirmed, but its effect on disability was not replicated [35]. Although the phase II and phase III studies both enrolled patients with RRMS and active disease, they differed mainly in the treatment history of the target populations (naive versus treatment failure). In a five-year follow-up of the phase II extension, the risk of sustained accumulation of disability from baseline to year 5 was reduced by $69 \%$ ( $p=$ $0.0005)$ in the AZB $12 \mathrm{mg}$ group compared to the IFN-beta-1a group [36]. Recent evidence has underlined the long-term beneficial effects of using AZB, with a very low proportion of $A Z B$-treated patients from the CARE-MS studies being found to progress from RRMS to SPMS (CARE-MS I, 1.1\%; CARE-MS II, 3.7\%) over six years of follow-up in the absence of continuous treatment. These results are, of course, based on defined criteria applied to the CARE-MS patient population, and they therefore require further confirmation in real-world cohorts [37].

The main safety issues with regard to the use of AZB concern the risk of developing of Graves disease and other adverse events that have been observed, such as Goodpasture syndrome, neoplasms and opportunistic infections.

AZB has been approved in Europe and Canada for highly active RRMS; more recently it has received regulatory approval from the Food and Drug Administration in the USA.

Up to know, 13.000 MS patients have been treated with AZB around the world gathering information from patients involved in aforementioned clinical trial extension and in real world clinical practice. These data confirmed how AZB reduces relapse rates, disability worsening, and the rate of brain volume loss over the long term. In a significant proportion of patients, preexisting disability remained stable or improved [38]. Nonetheless the present of safety issues such as infusion related reaction, development of autoimmunity caused by $\mathrm{T}$-cell repopulation occuring through reconstitution of T-cells in the thymus or proliferation of mature cells, infections have been confirmed. While infusion-associated reactions observed are quite manageable by clinicians, increased risk of infections (e.g: Listeria monocytogenes, herpes virus, cytomegalovirus), and secondary autoimmunity (e.g: idiopathic thrombocytopenic purpura, Graves's disease Goodpasture's syndrome) associated with AZB required a strict and rigorous monitoring even long after last treatment cycle [39]. To do so a clinical surveillance program for AZB has been established; protocol-defined laboratory monitoring including differential blood count, serum creatinine, and urine analysis before administration and monthly thereafter as well physician and patient education have been recommended continuing for 4 years after the last dose of AZB or longer if warranted [38]. This implies a precise patient selection relying on individual diligence and extreme compliance to the monitoring program.

Taking into consideration that AZB leads to significant long-lasting modification in the adaptive immunity cells and that no sufficient data on sequencing therapies after AZB are available to date to guide the clinician's choice, some authors suggest treating with first line medication (interferon or GA) those patients with breakthrough disease [15].

Cladribine is an adenosine deaminase-resistant purine nucleoside analog that preferentially reduces lymphocyte subpopulations. Cladribine pro-drug enters cells via purine nucleoside transporters and, once inside the cell, undergoes initial phosphorylation by deoxycytidine kinase. Lymphocytes are particularly susceptible to this effect because of their high intracellular ratio of deoxycytidine kinase to $5^{\prime}$-nucleotidase. The accumulation of cladribine nucleotides disrupts DNA synthesis and repair processes and ultimately leads to a sustained reduction in lymphocytes. Cladribine is able to cross the blood-brain barrier and is, therefore, likely to act on cells in both the periphery and the CNS; it appears to have a greater effect on CD4+ T-cells than on the CD8+ T-cell population [40].

Building on the experience with parenteral cladribine, a short-course of treatment with cladribine tablets was investigated for RRMS in the phase III CLARITY study [41]. The dosing regimen consisted of four courses of treatment during the first 48 weeks (weeks 1, 5, 9 and 13), followed by two further courses (starting at weeks 48 and 52). They received a cumulative dose of either 3.5 or $5.35 \mathrm{mg} / \mathrm{kg}$ over the 96 -week study, respectively). 
Treatment with cladribine tablets, 3.5 or $5.25 \mathrm{mg} / \mathrm{kg}$, significantly reduced the ARR at 96 weeks (relative reduction 57.6 and $54.5 \%$, respectively, vs placebo, both $p<0.001)$ and resulted in significantly more relapse-free patients at week 96 (79.7 and $78.9 \%$ vs $60.9 \%$, respectively; odds ratios: 2.53 and 2.43, both $\mathrm{p}<0.001$ ) [41].

ORACLE (ORAlCLadribine in Early $\underline{M S}$ ) was a 96-week, randomized, double-blind, placebo-controlled, international trial involving more than 616 clinically isolated syndrome patients. Cladribine, compared with placebo, significantly delayed the time to conversion to clinically definite MS; the authors reported a $62 \%$ risk reduction for $5.25 \mathrm{mg} / \mathrm{kg}$ dose (HR 0.38 ; 95\% CI $0.25-$ $0.58 ; p<0.0001$ ), and a $67 \%$ risk reduction for $3.5 \mathrm{mg} / \mathrm{kg}$ dose (HR 0.33; 95\% CI 0.21-0.51; $p<0.0001$ ). The high-dose cladribine treatment, versus placebo, resulted in a $91 \%$ risk reduction for new or persisting $\mathrm{T} 1$ Gd-enhancing lesions, and a 73\% risk reduction for new or enlarging T2 lesions [42]. During the open-label phase of the ORACLE study, all the participants who converted to definite MS were offered the possibility to continue on a twice-weekly maintenance therapy with IFN-beta-1a. The point estimate of the ARR in the open-label period was lower in patients originally randomized to cladribine tablets $3.5 \mathrm{mg} / \mathrm{kg}$ vs placebo in the double-blind treatment period [42]. In clinical trial settings, cladribine has shown a good tolerability profile; despite the lymphopenia that has been observed in cladribine-treated patients, the overall incidence of infections was similar across treatment groups. However, in the CLARITY study, herpes zoster infection was reported in eight patients in the cladribine $3.5 \mathrm{mg} / \mathrm{kg}$ group and in 12 patients in the $5.25 \mathrm{mg} / \mathrm{kg}$ group, and was inversely correlated with the lymphocyte counts $(p=0.003)$ [41]. Moreover the risk of cancer $(0.34 \%)$ was significantly higher in the cladribine than in the placebo groups [41]. However a meta-analysis of phase III trials of licensed DMDs for RRMS patients and CLARITY study aimed to compare the cancer risk associated with cladribine, assesed that the rate of cancer was similar between the phase III trial of cladribine and all other phase III trials of DMDs in RRMS patiens. Moreover a significant difference emerged when comparing the placebo groups of CLARITY (no cancers) with the placebo arms of all other phase III trials included in this study [43].

To better define the long-term safety profile of this drug, all patients previously enrolled in any clinical trial with cladribine are invited to join the PREMIERE (Prospective Observational Long-term Safety Registry of Multiple Sclerosis Patients Who Have Participated in Cladribine Clinical Studies) study. The follow-up will consist of over 10,000 patient years of exposure, in total, with follow-up in some patients exceeding eight years at completion (clinicatrial.gov: NCT01013350). The planned
End Date of the PREMIERE is established for November 2018. A first report of pooled safety data has been presented during the last American Academy of Neurology meeting held in Boston in April 2017. During a study period of 194 weeks (mean), as expected Lymphopenia was expected from cladribine tablets' mode of action, lymphopenia was reported more frequenly in cladribine treated patients than in placebo group. Consequentely herpes zoster was reported more frequently in patients experiencing Grade 3 or 4 lymphopenia; no clustering of types of malignancy, and no malignancies commonly associated with immunosuppression were observed during the follw-up period [44].

In July 2016, the European Medicines Agency accepted for review the marketing authorization application for cladribine tablets as a treatment for RRMS and its positive decision was stated in august 2017.

Since the compound has just been released on the market and given the relatively small population of patients in which cladribine has been used until now, more data are certainly warranted and future real world studies, along with clinician experience, will provide them accordingly. The results presented above, especially those regarding safety issues, need to be interpreted with caution due to the relatively short follow-up period. Despite these limitations, data derived from clinical trial on efficacy and safety as well the administration protocol and route are encouraging. Anyway the usage of IFN-beta-1a during the open label phase of ORACLE study has draft a possible treatment approach to be followed.

\section{Conclusions}

The range of possible treatments for MS has undergone a rapid expansion in recent years, and while this development certainly represents an enormous resource, on the other hand it presents every MS specialist with major challenges.

A considerable body of evidence supports the benefit of early treatment compared to delayed treatment; indeed, disability has been shown to continue to accumulate when therapies are started in the later disease stages.

The two case reports presented herein are paradigmatic in this regard. In case report 1 , the patient was considered, from the first observation, to be at high risk of disability progression, as she presented several negative prognostic factors. The clinician decided to start with immunosuppression as an induction treatment followed by a maintenance therapy with a first-line drug. The patient has remained stable over a long period of time.

The patient described in case report 2 presented several negative prognostic factors at disease onset, associated 
with a high risk of disease progression. In this case, first-line therapy was started despite several clinical and MRI parameters have already demonstrated a high disease activity. Thus, the induction therapy was then suggested with minimal advantages during the follow-up. So that a second line therapy was needed.

In this context two critical points should be underlined:

1. In highly active patients an induction treatment should be started as soon as possible.

The hypothesis that early treatment with high efficacy DMTs such as AZB or cladribine could result in better disease control and improved long term disease outcomes compared to the later commencement of high-efficacy DMTs is a crucial point to clarify in order to optimize the management of MS patients.

In a recent systematic review the timing of high-efficay therapy in relapsing remitting multiple sclerosis has been evaluated. The authors concluded that earlier treatment with high-efficacy, higher risk therapies for RR MS lead to a better control of relapse activity than their later initiation. However they also underlined that the evidence regarding the effect of the timing of high efficacy therapies on disability outcomes is conflicting and randomized clinical trials (RCT) or quality observation studies are warranted to answer this question [45].

2. Early identification of non-responders is crucial in order to allow the clinician to rapidly reach an optimal therapeutic decision with regard to the introduction of a more aggressive treatment.

Several papers proposed how to define and monitor response to MS treatments, and concluded that here is still no shared consensus on definition of treatment response. One important weakness when evaluating tools for assessing treatment response is that the vast majority of studies are based upon patients on IFNB and GA treatment, with limited data from cohorts of patients treated more effective drugs, such as AZB or cladribine. In our opinion, considering the lack of definition of treatment response for AZB and cladribine, the presence of disease activity occurring after immune cell-depletive agents, such as AZB or cladribine, can be contemplated as an indication to retreat the patient.

The decision to delay a more aggressive treatment is often based on safety concerns perceived by the clinician. Indeed, more aggressive treatments, as well as showing higher efficacy in controlling disease activity, are usually characterized by a higher risk profile because of their mechanisms of action. Nonetheless safety issues should not limit the use of these treatments; instead, clinicians need to strictly follow adequate monitoring programs and, especially, weigh up, on a patient-by-patient basis, the benefits versus the risk outcomes. In other words, even though definite clinical or radiological surrogate markers of disability prediction are not fully available as yet, clinicians need to evaluate the risk of serious adverse events in individual patients and identify those with a high risk of developing disability. This task will become easier when longer-term safety data, able to help neurologists make more robust decisions, become available for these new compounds too.

Unfortunately, since now we have few data about the better treatment alternatives after induction strategy when long-lasting effect of "inductive agent" on the immune system will be added to the a further "immunomodulation". A long term study has shown that a short (6-months) course of MTX followed by maintenance therapy with an immunomodulatory drug such as interferon beta or GA, brings about a rapid reduction in disease activity and subsequent sustained disease control $[19,21,46]$. No data are available about long-term maintenance treatment if induction is achieved with AZB, Cladribine or hematopoietic cell-stem transplantation and actual wider scenario of first line drugs needs to be considered. On the other hand, application of induction treatment paradigm in rheumatology has shown that, using modern synthetic disease modifying antirheumatic drugs suppression of rheumatoid activity can be a realistic option, even after discontinuation of drugs [3]. The absence of RCT and substantial real life data on this issue requires that clinician and patients need to share care decision matching the level of risk a patient is willing to accept and his prognostic factors.

In any case the initial treatment choice and its timing is crucial as suggested by other discipline experience and by the few data on MS, waiting for RCT or quality observation studies needed to answer this question.

\section{Abbreviations \\ (aHSCT): Intense immunosuppression followed by autologous hematopoietic stem cell transplantation; AZB: Alemtuzumab; CNS: Central nervous system; CSF: Cerebrospinal fluid; Cys: Cyclophosphamide; DMTs: Disease-modifying therapies; EDSS : Expanded Disability Status Scale; GA: Glatiramer acetate; Gd: Gadolinium; IFN: Interferon; IVMP: Intravenous methyl prednisolone; JC: John Cunningham; MP: Methylprednisolone; MRC: Medical Research Council; MRI: Magnetic resonance imaging; MS: Multiple sclerosis; MTX: Mitoxantrone; ON: Optic neuritis; RA: Rheumatoid arthritis; RCT: Randomized clinical trial; RR: Relapsing-remitting; RRMS: Relapsing-remitting multiple sclerosis; SP: Secondary progressive; SPMS: Secondary progressive multiple sclerosis}

Availability of data and materials

Data sharing not applicable to this article as no datasets were generated or analyzed during the current study.

Authors' contributions

$\mathrm{SR}, \mathrm{SP}$ and $\mathrm{CG}$ wrote the manuscript. CG and $\mathrm{CT}$ revised the manuscript. All authors read and approved the final manuscript.

Ethics approval and consent to participate Not applicable 


\section{Consent for publication}

Not applicable

\section{Competing interests}

SR has received fees as invited speaker or travel expenses for attending meeting from Biogen, Teva, Merk-Sereno. SP has received fees as invited speaker from Biogen, Teva, Sanofi, Novartis and Almirall. CT received honoraria for speaking from Biogen, Sanofi-Aventis. Teva, Bayer-Schering and Novartis. CG has received fees as invited speaker or travel expenses for attending meeting from Biogen, Merck-Serono, Teva, Sanofi, Novartis, Genzyme. Authors have nothing to disclose related to this manuscript.

\section{Publisher's Note}

Springer Nature remains neutral with regard to jurisdictional claims in published maps and institutional affiliations.

\section{Author details}

'Department of Human Neurosciences, "Sapienza" University of Rome, Viale dell'Università 30, 00185 Rome, Italy. ${ }^{2}$ Department of Neurosciences, S Camillo-Forlanini Hospital, Circonvallazione Gianicolense, 87, 00152 Rome, Italy

Received: 29 November 2017 Accepted: 20 July 2018

Published online: 14 August 2018

\section{References}

1. D'Amico E, Ziemssen T, Cottone S. Induction therapy for the management of early relapsing forms of multiple sclerosis. A critical opinion. Expert Opin Pharmacother. 2017;18:1553-6.

2. Pui C-H, Evans WE. Treatment of acute lymphoblastic leukemia. N Engl J Med. 2006;354:166-78.

3. Van Den Broek M, Lems WF, Allaart CF. BeSt practice: The success of earlytargeted treatment in rheumatoid arthritis. Clin Exp Rheumatol. 2012;30(4 SUPPL):73.

4. Pardo G, Jones DE. Correction to: the sequence of disease-modifying therapies in relapsing multiple sclerosis: safety and immunologic considerations. J Neurol. 2017;264:2375-7. https://doi.org/10.1007/s00415-017-8594-9.

5. Edan $\mathrm{G}$, Le Page E. Induction therapy for patients with multiple Sclerosis: Why? When? How? CNS Drugs. 2013;27(6):403-9.

6. McMahon EJ, Bailey SL, Castenada CV, Waldner H, Miller SD. Epitope spreading initiates in the CNS in two mouse models of multiple sclerosis. Nat Med. 2005;11:335-9.

7. Dendrou CA, Fugger L, Friese MA. Immunopathology of multiple sclerosis. Nat Publ Gr. 2015;15:545-58.

8. Leray E, Yaouang J, Le Page E, Coustans M, Laplaud D, Oger J, et al. Evidence for a two-stage disability progression in multiple sclerosis. Brain. 2010;133:1900-13.

9. Freedman MS. Induction vs. escalation of therapy for relapsing Multiple Sclerosis: The evidence. Neurol Sci. 2008:29(SUPPL. 2):250-2.

10. Ziemssen T, De Stefano N, Sormani MP, Van Wijmeersch B, Wiendl H, Kieseier BC. Optimizing therapy early in multiple sclerosis: an evidencebased view. Mult Scler Relat Disord. 2015:4:460-9.

11. Scalfari A, Neuhaus A, Degenhardt A, Rice GP, Muraro PA, Daumer M, et al. The natural history of multiple sclerosis, a geographically based study 10: relapses and long-term disability. Brain. 2010;133:1914-29.

12. Rush CA, Maclean HJ, Freedman MS. Aggressive multiple sclerosis: proposed definition and treatment algorithm. Nat Rev Neurol. 2015;11:379-89.

13. Menon S, Shirani A, Zhao Y, Oger J, Traboulsee A, Freedman MS, et al. Characterising aggressive multiple sclerosis. J Neurol Neurosurg Psychiatry. 2013:84:1192-8.

14. Fisniku LK, Brex PA, Altmann DR, Miszkiel KA, Benton CE, Lanyon R, et al. Disability and T2 MRI lesions: a 20-year follow-up of patients with relapse onset of multiple sclerosis. Brain. 2008:131:808-17.

15. Hassoun L, Eisele J, Thomas K, Ziemssen T. Hands on Alemtuzumabexperience from clinical practice: whom and how to treat. Mult Scler Demyelinating Disord. 2016;1:10

16. Cocco E, Marrosu MG. The current role of mitoxantrone in the treatment of multiple sclerosis. Expert Rev Neurother. 2014;14:607-16.

17. Edan G, Miller D, Clanet M, Confavreux C, Lyon-Caen O, Lubetzki C, et al. Therapeutic effect of mitoxantrone combined with methylprednisolone in multiple sclerosis: a randomised multicentre study of active disease using MRI and clinical criteria. J Neurol Neurosurg Psychiatry. 1997:62:112-8.

18. Le Page E, Leray E, Taurin G, Coustans M, Chaperon J, Edan G. Mitoxantrone as induction therapy in aggressive relapsing remitting multiple sclerosis: a descriptive analysis of 100 consecutive patients. Rev Neurol. 2006;162:185-94.

19. Edan G, Comi G, Le Page E, Leray E, Rocca MA, Filippi M. Mitoxantrone prior to interferon beta-1b in aggressive relapsing multiple sclerosis: a 3-year randomised trial. J Neurol Neurosurg Psychiatry. 2011;82(12):1344-50.

20. Vollmer T, Panitch H, Bar-Or A, Dunn J, Freedman M, Gazda S, et al. Glatiramer acetate after induction therapy with mitoxantrone in relapsing multiple sclerosis. Mult Scler J. 2008;14:663-70.

21. Le Page E, Leray E, Edan G, Grp FMS. Long-term safety profile of mitoxantrone in a French cohort of 802 multiple sclerosis patients: a 5-year prospective study. Mult Scler J. 2011;17:867-75.

22. Martinelli V, Cocco E, Capra R, Salemi G, Gallo P, Capobianco M, et al. Acute myeloid leukemia in Italian patients with multiple sclerosis treated with mitoxantrone. Neurology. 2011;77(21):1887-95.

23. Stroet A, Hemmelmann C, Starck M, Zettl U, Dörr J, Friedemann P, et al. Incidence of therapy-related acute leukaemia in mitoxantrone-treated multiple sclerosis patients in Germany. Ther Adv Neurol Disord. 2012;5:75-9.

24. Awad A, Stüve O. Review: cyclophosphamide in multiple sclerosis: scientific rationale, history and novel treatment paradigms. Ther Adv Neurol Disord. 2009:2:357-68

25. Patti F, Lo FS. Lights and Shadows of Cyclophosphamide in the Treatment of Multiple Sclerosis. Autoimmune Dis. 2011;2011:961702.

26. Harrison DM, Gladstone DE, Hammond E, Cheng J, Jones RJ, Brodsky RA, et al. Treatment of relapsing - remitting multiple sclerosis with high-dose cyclophosphamide induction followed by glatiramer acetate maintenance. Mult Scler. 2012;18(2):202-9.

27. Weinstock-Guttman B, Kinkel RP, Cohen JA, Ransohoff RM, Schwetz K, Gogol D, et al. Treatment of fulminant multiple sclerosis with intravenous cyclophosphamide. Neurologist. 1997:3:178-85.

28. De Ridder D, van Poppel H, Demonty L, D'Hooghe B, Gonsette R, Carton $\mathrm{H}$, et al. Bladder cancer in patients with multiple sclerosis treated with cyclophosphamide. J Urol. 1998;159:1881-4.

29. Sola-Valls N, Sepúlveda M, Blanco Y, Saiz A. Current role of chemotherapy and bone marrow transplantation in multiple sclerosis. Curr Treat Options Neurol. 2015;17:1-20.

30. Reston JT, Uhl S, Treadwell JR, Nash RA, Schoelles K. Autologous hematopoietic cell transplantation for multiple sclerosis: a systematic review. Mult Scler. 2011; 17:204-13.

31. Mancardi GL, Sormani MP, Gualandi F, Saiz A, Carreras E, Merelli E, et al. Autologous hematopoietic stem cell transplantation in multiple sclerosis: a phase II trial. Neurology. 2015;84:981-8.

32. Dörr J, Baum K. Alemtuzumab in the treatment of multiple sclerosis: patient selection and special considerations. Drug Des Devel Ther. 2016;10:3379-86.

33. CAMMS223 Trial Investigators, Coles AJ, Compston DAS, Selmaj KW, Lake SL, Moran S, et al. Alemtuzumab vs. interferon beta-1a in early multiple sclerosis. N Engl J Med. 2008:359:1786-801.

34. Coles AJ, Fox E, Vladic A, Gazda SK, Brinar V, Selmaj KW, et al. Alemtuzumab versus interferon beta-1a in early relapsing-remitting multiple sclerosis: post-hoc and subset analyses of clinical efficacy outcomes. Lancet Neurol. 2011;10:338-48.

35. Cohen JA, Coles AJ, Arnold DL, Confavreux C, Fox EJ, Hartung H-P, et al. Alemtuzumab versus interferon beta 1a as first-line treatment for patients with relapsing-remitting multiple sclerosis: a randomised controlled phase 3 trial. Lancet. 2012;380:1819-28.

36. Coles AJ, Fox E, Vladic A, Gazda SK, Brinar V, Selmaj KW, et al. Alemtuzumab more effective than interferon??-1a at 5-year follow-up of CAMMS223 clinical trial. Neurology. 2012;78:1069-78.

37. Horakova D, Boster A, Ziemssen T, Bertolotto A, Goelz SE, Firmino I, et al. Patients Who Received Alemtuzumab in CARE-MS I or II Show a Low Rate of Conversion From Relapsing-Remitting MS to Secondary Progressive MS Through 6 Years 69 th Annual Meeting of the American Academy of Neurology ( AAN ) Patients Who Received Alemtuzumab. AAN 2017. 2017;:P4(407):6-7.

38. Ziemssen T, Thomas K. Alemtuzumab in the long-term treatment of relapsingremitting multiple sclerosis: an update on the clinical trial evidence and data from the real world. Ther Adv Neurol Disord. 2017:10:343-59.

39. Guarnera C, Bramanti P, Mazzon E. Alemtuzumab: a review of efficacy and risks in the treatment of relapsing remitting multiple sclerosis. Ther Clin Risk Manag. 2017;13:871-9 
40. Liliemark J. The clinical pharmacokinetics of cladribine. Clin Pharmacokinet. 1997;32:120-31.

41. Giovannoni G, Comi G, Cook S, Rammohan K, Rieckmann P, Sorensen PS, et al. A placebo-controlled trial of oral Cladribine for relapsing multiple sclerosis. N Engl J Med. 2010;362(5):416-26.

42. Comi G, Leist T, Freedman MS, BAC C, Coyle PK, Vermersch P, et al. Cladribine tablets in the ORACLE-MS study open-label maintenance period: analysis of efficacy in patients after conversion to clinically definite multiple sclerosis (CDMS). Neurol. 2017;88(Supplement 16)(P6.349).

43. Pakpoor J, Giulio Disanto B, Altmann DR, Sue Pavitt F, Turner BP, Monica Marta $F$, et al. No evidence for higher risk of cancer in patients with multiple sclerosis taking cladribine. Neurol Neuroimmunol Neuroinlamm. 2015;2:1.

44. Cook S, Leist T, Comi G, Montalban X, Sylvester E, Hicking C, et al. Cladribine tablets in the treatment of patients with multiple sclerosis (MS): an integrated analysis of safety from the MS clinical development program. Neurol. 2017;88(Supplement 16)(P5.394).

45. Merkel B, Butzkueven H, Traboulsee AL, Havrdova E, Kalincik T. Timing of high-efficacy therapy in relapsing-remitting multiple sclerosis: a systematic review. Autoimmun Rev. 2017;16(6):658-65.

46. Le Page E, Leray E, Taurin G, Coustans M, Chaperon J, Morrissey SP, et al. Mitoxantrone as induction treatment in aggressive relapsing remitting multiple sclerosis: treatment response factors in a 5 year follow-up observational study of 100 consecutive patients. J Neurol Neurosurg Psychiatry. 2008;79:52-6.

Ready to submit your research? Choose BMC and benefit from:

- fast, convenient online submission

- thorough peer review by experienced researchers in your field

- rapid publication on acceptance

- support for research data, including large and complex data types

- gold Open Access which fosters wider collaboration and increased citations

- maximum visibility for your research: over $100 \mathrm{M}$ website views per year

At $\mathrm{BMC}$, research is always in progress.

Learn more biomedcentral.com/submissions 